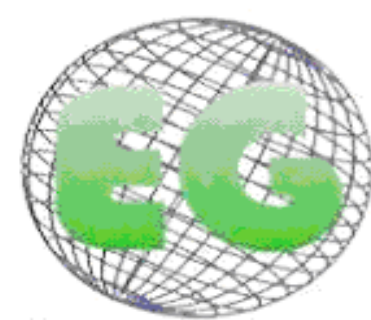

ISSN 1695-6141 No22
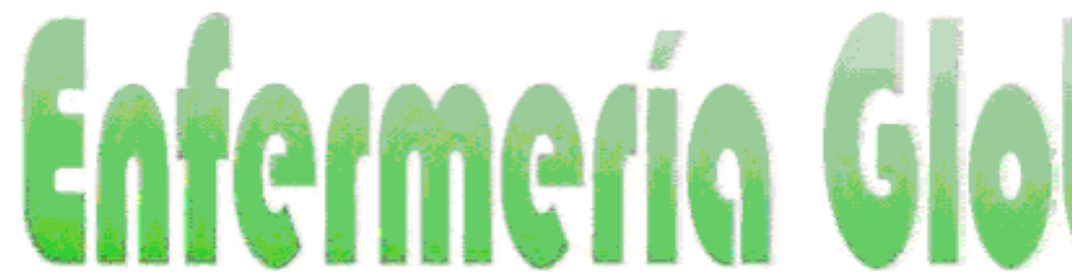

Revista electrónica trimestral de Enfermería

www.um.es/egloball

Abril 2011

\title{
PROGRAMA PARA CUIDADORES DE ANCIANOS CON DEMENCIA:UN RELATO DE EXPERIENCIA
}

PROGRAMA PARA CUIDADORES DE IDOSOS COM DEMÊNCIA: UM RELATO DE EXPERIÊNCIA

*Da Costa Lindolpho, M., "*Bum, AK., ${ }^{* * *}$ Chaves Sá, SP., "***Peçanha da Cruz, TJ., ***** Silva de Andrade, F.

*Mestre em Enfermagem. Professora Adjunta da Escola de Enfermagem Aurora de Afonso Costa da Universidade Federal Fluminense- EEAAC/UFF e Coordenadora do PRO-CUIDEM. **Doutora em Enfermagem. Professora da Escola de Enfermagem Alfredo Pinto da Universidade do Rio de JaneiroEEAP/UNIRIO e Vice-coordenadora do PRÓ-CUIDEM. ${ }^{* * *}$ Doutora em Enfermagem. Professora Titular da Escola de Enfermagem Aurora de Afonso Costa Universidade Federal Fluminense-EEAAC/UFF. ****Enfermeira. Bolsista PIBIC do projeto Estimulação Cognitiva com idosos portadores de demência - uma proposta do Programa de Enfermagem Gerontogeriátrico da UFF no período de $2008-2009 .{ }^{* * * * *}$ Enfermeira. Bolsista de extensão do Pró-Cuidem em 2009.

Palabras clave: Anciano; Demencia; Cuidadores
Palavras-chave: Idoso; Demência; Cuidadores.

Keywords: Elderly; Dementia; Carers.

\section{RESUMEN}

El cuidar del anciano con demencia en el día a día es una tarea muy difícil cuando no se tiene soporte y orientación. Pensando en este aspecto se creó el PRÓ-CUIDEM, un grupo de orientación al cuidador que forma parte del Proyecto de Extensión a Enfermería en el Programa Interdisciplinar de Geriatría y Gerontología (EPIGG), realizado en la Universidad Federal Fluminense (UFF), en Niterói-RJ. Este grupo tiene el objetivo de acoger a este cuidador y orientarlo sobre la mejor forma de atender las necesidades, principalmente de autocuidado del anciano con esa enfermedad, minimizando los trastornos a ambos. Al mismo tiempo, contribuye a un intercambio de experiencias entre los cuidadores, minimizando los conflictos y aprendiendo diferentes formas de cuidar disminuyendo el estrés. Se trata de un relato de experiencia sobre el grupo, realizándose cuatro encuentros mensuales, con duración aproximada de dos horas. Estas reuniones tienen lugar una vez por semana y son coordinadas por enfermeras docentes con participación de alumnos de enfermería. De este estudio se concluye que los cuidadores necesitan hoy principalmente de una atención mayor y es a través de este grupo que sus dudas son aclaradas lo que hace que se sientan menos 
ansiosos, más dispuestos a cuidar del anciano con comprensión y principalmente cuidar de su propia salud.

\section{RESUMO}

O cuidar do idoso com demência no dia a dia torna-se uma tarefa muito difícil quando não se tem suporte e orientação. Foi pensando neste aspecto que se criou o PRÓ-CUIDEM, um grupo de orientação ao cuidador. Sendo parte do Projeto de Extensão a Enfermagem no Programa Interdisciplinar de Geriatria e Gerontologia (EPIGG), realizado na Universidade Federal Fluminense (UFF), em Niterói-RJ. Este grupo tem o objetivo de acolher esse cuidador e orientá-lo sobre a melhor forma de atender as necessidades, principalmente de autocuidado do idoso com essa doença, minimizando os transtornos a ambos. Ao mesmo tempo, contribui para uma troca de experiência entre os cuidadores, amenizando os conflitos e conhecendo diferentes formas de cuidar diminuindo o estresse. Trata-se de um relato de experiência sobre o grupo, onde são realizados quatros encontros mensais, com duração de aproximadamente duas horas cada. Esses encontros ocorrem uma vez por semana e são coordenados por enfermeiras docentes com participação dos alunos da enfermagem. Deste estudo conclui-se que os cuidadores necessitam principalmente nos dias de hoje, de uma atenção maior e é através desse grupo que suas dúvidas são esclarecidas e faz com que eles se sintam menos ansiosos, mais dispostos a cuidar do idoso com compreensão e principalmente cuidar da sua própria saúde.

\section{ABSTRACT}

The daily care of the elderly with dementia, becomes a very difficult task when there is no support and guidance. Based on this aspect, a group to support the carers was created (PRÓ-CUIDEM). This Program is part of the Extension Project: the Nursery in the Interdisciplinary Program of Geriatry and Gerontology (EPIGG) at the Universidade Federal Fluminense (UFF), in Niterói-RJ. This group was set up to receive carers, to guide them in the best way to care for the needs of the elderly and principally to take care of themselves and minimize disturbances to both. At the same time, this program contributes as an exchange of experiences among the carers, brightening up the conflicts and learning about different ways of how to take care of them, so reducing stress. It is an experience report about the group, based on meetings that are held four times a month, with a duration of approximately two hours. These meetings occur once a week and are coordinated by teaching nurses with the participation of nursing students. It can be concluded that through these meetings, carers can clarify doubts, become less anxious and more willing to take care of themselves and the elderly.

\section{INTRODUCCIÓN}

Hoy en Brasil se estima que existen cerca de 17,6 millones de ancianos ${ }^{(1)}$. El envejecimiento de la población y el aumento de la expectativa de vida, cuando se resiste al envejecimiento fisiológico, trae como consecuencia la aparición de dolencias crónico-degenerativas, entre las que sobresalen las demencias ${ }^{(2)}$.

Para Filho \& Netto ${ }^{(3)}$ la demencia se entiende como un síndrome que afecta al raciocinio, a la memoria, a la percepción, a la atención, a la capacidad de reconocer, al lenguaje y a la personalidad. Ellos también afirman que "la prevalencia de demencia se duplica cada cinco años después de los 65 años, aumentando exponencialmente con la edad."

Son varios los tipos y causas de demencia. Por eso es importante el diagnóstico precoz para escoger el mejor tratamiento. Algunas son reversibles como: toxicidad de medicamentos, depresión, infección del sistema nervioso, hematomas subdurales, tumores cerebrales primarios, hidrocefalia de presión normal, envenenamiento orgánico y metálico, disfunciones de tiroides y paratiroides, y deficiencias nutricionales como B12, B6, tiamina y ácido fólico $^{(4)(5)}$. 
Otras son irreversibles, como la demencia vascular, demencias de los cuerpos de Lewy, las demencias frontotemporales -específicamente la enfermedad de Pick- y la enfermedad de Alzheimer, siendo esta última considerada el tipo de demencia más frecuente (de 50 a $65 \%$ de los casos) entre los ancianos. Son estas las que necesitan de cuidados continuos ${ }^{(4)(5)(6)}$.

La pérdida progresiva de la memoria es una característica en la demencia, además el síndrome demencial incluye al menos una de las siguientes pérdidas cognitivas: afasia; agnosia; apraxia y alteración de las funciones de ejecución, como planificación, organización, secuencia y abstracción ${ }^{(2)}$.

Según el grado de evolución de la demencia, la pérdida de las capacidades funcionales (déficit de memoria, percepción y coordinación motora) es lo que más afecta al individuo, comprometiendo principalmente sus condiciones para realizar el autocuidado.

Para Sá et. al. ${ }^{(7)}$ el anciano con demencia "desarrolla una relación extrema de dependencia con su cuidador" lo que genera angustia, pues pierde autonomía para realizar determinadas actividades, "además de ser algo que requiere gran confianza y seguridad" en el otro.

Al hacerse los ancianos cada vez más dependientes, los trastornos demenciales "pueden provocar sufrimiento tanto para los ancianos como para sus familiares, que son obligados a reorganizarse para viabilizar los cuidados a la persona enferma"(7).

Como esa confianza sólo existe con personas que ya se conocen tiempo, con la que hay una relación de compañerismo, amistad y respeto, la mayoría de las veces quien asume los cuidados con el anciano es el miembro más próximo de la familia. Pero incluso con tamaña afinidad que esa persona tiene con el anciano, no se siente preparada para asumir los cuidados directos al enfermo.

Cuando el diagnóstico es una realidad, los profesionales de la salud buscan proporcionar condiciones para que los cuidadores lo afronten y el primer paso es informar a los mismos de las características básicas de la enfermedad. Al obtener información sobre la enfermedad, su evolución, los diversos tipos de enfrentamientos, de las dificultades y los desafíos, este sufrimiento se reduce ${ }^{(8)}$. Y para ello, la ayuda de profesionales especializados, en varios niveles de atención, es categórica para la determinación del bienestar del cuidador y de la calidad de los cuidados prestados a los enfermos con demencia ${ }^{(9)}$.

En este sentido, las oficinas terapéuticas y grupos de orientación son de extrema valía. De acuerdo con Sá et al. ${ }^{(7)}$, es preciso estimular a los cuidadores a participar de las oficinas y reconocer la importancia del apoyo social formal e informal. Esto también contempla el ámbito de la acción del grupo de apoyo.

El cuidador, de acuerdo con el decreto $n^{\circ} 1395 / \mathrm{gm}$ de 10/12/1999, es toda persona, miembro o no de la familia, que con o sin remuneración, formal o informal (cuidador formal es todo aquel que es profesional y utiliza las técnicas aprendidas en el cuidado al anciano y cuidador informal es aquel que no es profesional, generalmente son los familiares, amigos y compañeros), presta cuidado al anciano que depende de auxilio en sus actividades diarias, como: alimentación, higiene personal, medicación, acompañamiento a los servicios de salud, servicios de bancos o farmacias entre otros ${ }^{(10)}$. 
De esa forma el Decreto afirma que "estas personas deberán, también, recibir atención médica personal, considerando que la tarea de cuidar de un adulto dependiente es desgastante e implica riesgos para la salud del cuidador ${ }^{(10)}$."

Con todo, en todas las áreas en que el anciano es atendido, se observa el crecimiento del número de ancianos con demencia. De ahí la necesidad de formación de más espacios informativos, como los grupos de apoyo para orientación de las personas que cuidan de estos ancianos con demencia. Tal hecho, nos remite a recordar la importancia del enfermero en esa atención, teniendo como primordial, la busca de alternativas y maneras capaces de minimizar el impacto de esta enfermedad en la vida de la familia/cuidador.

Por ello, incluso con la gran experiencia presentada por esa persona en cuidar del otro, no siempre es suficiente para lidiar con el anciano demenciado, incluso porque debido a factores emocionales, los familiares acaban por presentar dificultades con ese cuidado, ya que inicialmente ellos pasan por un momento de aceptación de la enfermedad. Así, de acuerdo con Néri \& Carvalho, se observa que "la ayuda de un profesional especializado [...] es crucial para la determinación del bienestar del cuidador y principalmente de los cuidados prestados a los ancianos ${ }^{(9)}$."

A partir de esta realidad, se creó el Programa para Cuidadores de Idosos com Demência (PRÓ-CUIDEM) dentro del Projeto de Extensão "A Enfermagem no Programa Interdisciplinar de Geriatria e Gerontologia da Universidade Federal Fluminense"- (EPIGG/UFF), con la finalidad de ofrecer informaciones, orientaciones y soporte a los cuidadores acerca de la patología, terapéutica y sus cuidados. Así, el objetivo de este artículo es describir las experiencias realizadas a lo largo de las reuniones del PRÓ-CUIDEM.

\section{METODOLOGÍA}

Se trata de un relato de experiencia sobre el grupo de orientación para cuidadores de ancianos com demencia realizado en el Projeto de Extensão EPIGG realizado en la Universidad Federal Fluminense (UFF), estado de Rio de Janeiro.

\section{EI PRÓ-CUIDEM}

EI PRÓ-CUIDEM surgió a través de la articulación entre una docente de Enfermería de la Universidad de Rio de Janeiro (UNIRIO), con dedicación parcial en el Programa Interdisciplinar de Geriatría y Gerontología de la UFF con las docentes de la Escuela de Enfermería de la UFF que ya desarrollaban sus actividades en el EPIGG. EI Proyecto fue construido y encaminado al Decano de Extensión de la Universidad Federal Fluminense, contemplándose con dos becarios. Este programa complementó las actividades del EPIGG dirigiéndose a las necesidades del cuidador.

EI EPIGG/UFF es un proyecto de extensión que está incluido en el Programa Interdisciplinar de Geriatría y Gerontología de la UFF. Este programa, con profesionales de diversas áreas, atiende ancianos sanos o con enfermedades crónicas y degenerativas, siendo también una referencia en la asistencia al anciano con demencia y sus cuidadores.

Con el aumento considerable de atención a ancianos con demencia y sus cuidadores en el EPIGG/UFF en consulta de enfermería, las docentes observaron la falta de información sobre la enfermedad, el miedo de los cuidadores ante el diagnóstico y la falta de preparación para el cuidado al anciano portador de demencia. Así, el PRÓ-CUIDEM fue elaborado como 
actividad abierta a los cuidadores de ancianos con demencia que son atendidos en el programa de la universidad o no.

Se abren mensualmente 20 (veinte) vacantes para inscripción de cuidadores que deseen participar del Pró-cuidem, que se caracteriza por 4 (cuatro) encuentros al mes, los lunes, con una duración aproximada de 2 horas cada uno. En estos encuentros semanales tienen lugar conferencias coordinadas por enfermeras con la participación de becarios de extensión, de iniciación científica y alumnos de graduación de enfermería. En cada ciclo, el grupo de cuidadores está formado por un máximo de hasta 20 personas, para que todos los participantes puedan relatar sus experiencias y así promover una mayor interacción unos con otros y facilitar la intervención del profesional cuando hubiera dudas para ser aclaradas.

EI PRÓ-CUIDEM tiene como objetivos: informar a los cuidadores de ancianos sobre las alteraciones del proceso demencial y de la terapéutica recomendada; orientar a los cuidadores de ancianos con demencias sobre los cuidados diarios en el domicilio; promover la salud del cuidador y del anciano con demencia frente a las necesidades en los cuidados diarios y presentar las medidas y recursos posibles para que haya una estimulación cognitiva de ese anciano en el propio ambiente familiar.

Sus estrategias pedagógicas son aulas dialogadas; dinámicas de grupo; folletos informativos y filmes.

Los cuatro encuentros de cada ciclo de orientaciones poseen los siguientes contenidos programáticos:

- Nociones básicas del proceso demencial: en este módulo son presentados los tipos de demencia, las 10 señales más comunes de la demencia, las patologías que cursan con enfermedad, la enfermedad de Alzheimer, las etapas de la demencia.

- Nociones básicas de medicación: son presentados los tipos de fármacos utilizados, sus finalidades, su administración, reacciones adversas más comunes y los cuidados básicos.

- Cuidados diarios frente a los desafíos del proceso demencial, donde se preveían orientaciones acerca del cuidado al anciano en el domicilio, en las vestimentas, en el baño, en la higiene, en la promoción del autocuidado, las alternativas encontradas y sugeridas para la mejor convivencia de este anciano, no olvidando nunca la seguridad proporcionada por el ambiente.

- Nociones básicas de la estimulación cognitiva: ante la necesidad de mantener activa la mente del anciano y retardar el avance de la demencia, en esa aula se prevén sugerencias de cómo estimular al anciano en su domicilio. Instrumentos comunes de nuestro día a día son presentados y explicados cómo deben ser utilizados en la situación de tener un anciano en proceso demencial en casa.

Antes de iniciar los encuentros, la sala es preparada y las sillas son ordenadas de acuerdo con la temática (o en círculos, o de frente para el lugar en que será proyectado en el aula). En el primer encuentro, la enfermera docente, juntamente con las estudiantes becarias, realizan una ronda de conversación preguntando cómo fue la semana con el anciano, como estaban sabiendo del PRÓ-CUIDEM. En ese momento, los cuidadores tienen la oportunidad de contar las experiencias, dificultades y satisfacciones vividas en la última semana. A partir de ahí, transcurren las otras actividades programadas. 


\section{RESULTADOS Y DISCUSIONES}

Al final, realizamos treinta y ocho encuentros, formando nueve grupos en total en el período de marzo a diciembre de 2008. Las expectativas de cada integrante del grupo fueron emergiendo y los cuidadores se identificaban, señalando las semejanzas y lo que reconocían en su anciano en el cotidiano. Los lapsos de memoria, comportamientos inadecuados son los puntos más comentados. Con el intercambio de experiencias, un cuidador pasa a otro, intermediado por la docente, sus frustraciones, desafíos, alegrías y sugerencias para el enfrentamiento de la enfermedad y de las situaciones que surgen con ella.

Otro punto também observado es que los cuidadores en la mayoría de los grupos eran predominantemente del sexo femenino, "la mujer se evidencia como 'gran cuidadora'”, ya que siempre le fue atribuido ese papel de cuidar de los hijos, del marido, de los enfermos y consecuentemente de los "viejos ${ }^{(11)}$."

Hay también un porcentaje considerable de ancianos cuidando de ancianos, lo que limita la calidad del cuidado prestado al anciano demenciado, pues este anciano/cuidador ya no se encuentra en condiciones físicas y hasta incluso emocionales de asumir el cuidado de ese otro anciano.

Se observó una carencia muy grande en esos cuidadores de informaciones, principalmente aquellas que están relacionadas con la fase de descubrimiento de la dolencia, sobre el tratamiento más indicado y cuáles son las mejores estrategias para lidiar con ese tipo de anciano en el día a día.

Cada encuentro es iniciado con una rueda de conversaciones, buscando de los participantes cuáles son sus principales cuestionamientos, evaluando cuál es el nivel de información que cada cuidador posee ante la enfermedad y cuidados esenciales que se deben tener con el anciano en cuestión.

En ese momento se produce un riquísimo intercambio de experiencias, donde los cuidadores y familiares escuchan y hablan de sus vivencias con el anciano en proceso demencial, de sus angustias y dificultades en el cuidar, siendo una demostración rica de solidaridad y de compasión con las dificultades de cada uno.

Asiduidad y gran aceptación de los temas sugeridos en cada encuentro fue lo observado en todos los grupos que pasaron por el PRÓ-CUIDEM.

La participación de los cuidadores en el grupo de orientación, según sus propios relatos, disminuyó la sensación de sentirse solo, vivenciando esa situación embarazosa.

Al final de cada grupo, se distribuyó un formulario para que los participantes puedan exponer su evaluación y sugerencias acerca del contenido, conferenciantes y demás aspectos abordados. Fueron completados solo 54 formularios, por lo que calculamos tener aproximadamente 108 participantes (media de 12 cuidadores en cada grupo), de lo que se deduce que hay un número inferior de formularios rellenados en comparación con el número de participantes. Aunque debe señalarse que la presencia de estos participantes no era obligatoria en todos los encuentros, lo que justifica la falta de formularios completados.

De estos 54 formularios de evaluación completados: 81,48\% eran cuidadores de sexo femenino, $9,25 \%$ de sexo masculino, $9,25 \%$ no respondieron el sexo, lo que confirma la 
observación citada en parágrafo anterior sobre el predominio del sexo femenino. En relación al contenido: $87,03 \%$ consideraron el contenido ÓPTIMO y 12,97\% consideraron BUENO, y ninguno indicó el contenido REGULAR o MALO. En cuanto a los profesores que impartieron las conferencias: 90,74\% consideraron a los profesores ÓPTIMOS; 9,26\% consideraron BUENOS, y ninguno indicó REGULAR o MALO. Sobre la forma de transmitir la información: $85,2 \%$ consideran ÓPTIMO y 14,8\% BUENO. En relación al item que preguntaba si los encuentros atendieron a las necesidades: $88,8 \%$ respondieron que atendieron TOTALMENTE; 9,35\% respondieron que atendió PARCIALMENTE y $1,85 \%$ no respondieron, y ninguno respondió que NO ATENDIÓ.

\section{CONCLUSIÓN}

Al crecer cada vez más la población de ancianos, con ella también aumenta el número de enfermedades crónicas y degenerativas como la demencia, pudiendo volver al anciano dependiente, necesitando del auxilio de cuidadores.

Se sabe que para realizar este cuidado, la persona debe obtener informaciones suficientes acerca de la enfermedad/tratamiento y un soporte emocional muy grande. Hay una creciente necesidad de orientar a los cuidadores de estos ancianos respecto al progreso de la enfermedad, sus manifestaciones, las complicaciones y lo que puede esperarse de un anciano con demencia.

Se percibió cómo es importante el trabajo realizado por el grupo de orientación a los cuidadores de ancianos con demencia - PRÓ-CUIDEM. Es un espacio en donde hay intercambio de saberes, tanto entre los profesionales, como del participante para el profesional y viceversa.

EI PRÓ-CUIDEM consiguió proporcionar informaciones acerca de las características peculiares de la demencia y lo que el cuidador precisa saber, mostrando que por detrás de un proceso demencial, existe un anciano que necesita de cuidados y que no puede ser considerado como un estorbo. Además, resulta evidente que detrás de un cuidador, existe un ser humano con problemas, frustraciones, alegrías e incertidumbres como cualquier otro en el mundo.

Con este trabajo, muchos cuidadores recibieron orientaciones y divulgaron a otros que se encuentran en la misma situación, mejorando la calidad de vida de los cuidadores, mejorando el cuidado prestado al anciano y, sobre todo, desmitificando la demencia.

Con todo, divulgar las acciones ejecutadas en este programa es permitir que siga discutiéndose la importancia de prestar un soporte a los cuidadores de ancianos con demencia, pues contribuyó a aliviar los problemas y favorecer el bienestar del cuidador y de quién está siendo cuidado. De esa forma también se contribuyó a una calidad en el cuidado al anciano.

Como una alerta para las políticas de atención a los ancianos, deseamos que esa actividad estimule la creación de más espacios en diversas instituciones, para la realización de esos grupos. Permitiendo la realización de un trabajo multiprofesional, cubriendo así todas las formas de tratamiento para los participantes. Pues cuando existen profesionales con visiones diferentes sobre una determinada situación, ellos pueden mezclarse y atender las necesidades del individuo de forma más completa. 
Se concluye que este programa es fundamental, pues proporciona al cuidador comprender lo que acontece con su familiar y obtener orientaciones para el cuidado al anciano con demencia. Los cuidadores necesitan, principalmente, en el día de hoy, de una atención mayor y es a través de este grupo que sus dudas son aclaradas y hace que ellos sientan placer nuevamente en vivir, más dispuestos a cuidar del anciano con demencia con comprensión y principalmente cuidar de su propia salud e insertarse en grupos de cuidadores para que puedan proseguir con un cuidado al anciano con más calidad y tranquilidad más allá de mejorar su trayectoria en este momento difícil que enfrenta.

\section{REFERENCIAS BIBLIOGRÁFICAS}

(1) IBGE. Ministério do Planejamento, Orçamento e Gestão. Contagem da população 2007. Rio de Janeiro, 2007. 311p. Disponível em: $<$ http://www.ibge.com.br/home/estatistica/populacao/contagem2007/contagem.pdf>. Acesso em: 14/09/2007.

(2) Machado JCB. Doença de Alzheimer. In: Freitas EV et al. Tratado de Geriatria e Gerontologia. Rio de Janeiro: Guanabara Koogan; 2000. p. 133-47

(3) FILHO ETC, NETTO MP. Geriatria Fundamentos, Clínica e Terapêutica. 2ª Ed. São Paulo: Atheneu. 2006, p. 788.

(4) Caldeira APS, Ribeiro RCHM. O enfrentamento do cuidador do idoso com Alzheimer. Arq Ciênc Saúde 2004 abr-jun;11(2):100-4

(5) Brasil. Ministério da Saúde. Série A: Normas e Manuais Técnicos: Envelhecimento e saúde da pessoa idosa. Caderno de atenção básica no 19. Brasília, 2006. p. 192.

(6) Caramelli P, Barbosa MT. Como diagnosticar as quatros causas mais freqüentes de demência? Rev. Bras. de Psiquiatr 2002,24(supl I):7-10.

(7) SÁ et.al. Oficinas terapêticas para cuidadores de idosos com demência-atuação da enfermagem no programa interdisciplinar de geriatria e gerontologia da UFF. Rev. Bras. de Geriatria e Gerontologia.UnATI/UERJ. Vol.9 N³. Set.-dez./2006. p.101-114.

(8) Guerreiro T, Caldas CP. Memória e demência: (re)conhecimento e cuidado. Rio de Janeiro: UERJ: UNATI, 2001.

(9) Neri AL, Carvalho UAML. O bem-estar do cuidador - aspectos psicossociais. In: FREITAS, E. U. et al. Tratado de geriatria e gerontologia. Rio de Janeiro: Guanabara Koogan, 2002, p. 778-89

(10) Portaria no 1395/GM de 10 / 12 / 1999. Política Nacional de Saúde do Idoso.

(11) DIOGO MJD, CEOLIM MF, CINTRA FA. Orientações para idosas que cuidam de idosos em domicílio. Rev. Esc. Enferm. USP.v.39 n¹. São Paulo. Março/2005.p.97-102. 
ISSN 1695-6141

(C) COPYRIGHT Servicio de Publicaciones - Universidad de Murcia 